\title{
Impact of thermal expansion coefficient on the local tilt angle of extreme ultraviolet pellicle
}

\author{
Junghwan Kim $\odot$, Haneul Kim, and Jinho Ahn* \\ Hanyang University, Department of Material Science and Engineering, Seoul, \\ Republic of Korea
}

\begin{abstract}
Background: A local tilt angle of $<300 \mathrm{mrad}$ results in a critical dimension uniformity (CDU) impact below $0.1 \mathrm{~nm}$ when a pellicle is used for extreme ultraviolet (EUV) lithography. However, the thermomechanical property guidelines satisfying this specification have not yet been established.
\end{abstract}

Aim: We present the thermomechanical property guidelines that yield a CDU impact below $0.1 \mathrm{~nm}$.

Approach: The peak temperature ranges of the EUV pellicle, as a function of the emissivity, were calculated through experimental, numerical, and finite element method analyses. The wrinkle profiles were evaluated as a function of the coefficient of thermal expansion (CTE) within these temperature ranges. The emissivity and CTE values satisfying the specifications were obtained using the CDU impact caused by the wrinkled EUV pellicle.

Results: The wrinkle amplitude in the EUV pellicle exhibited $45 \%$ attenuation with a twofold decrease in the CTE. The maximum local tilt angles for the 17, 16, and $15 \mathrm{~nm}$ half-pitch patterns were 290.2, 286.1, and $272.3 \mathrm{mrad}$, respectively. CTE below $2 \times 10^{-5} \mathrm{~K}^{-1}$ and emissivity above 0.1 are suggested for the EUV pellicle.

Conclusions: The CTE and emissivity guidelines satisfying the CDU impact specifications can be used for developing next-generation EUV pellicles.

(C) The Authors. Published by SPIE under a Creative Commons Attribution 4.0 International License. Distribution or reproduction of this work in whole or in part requires full attribution of the original publication, including its DOI. [DOI: 10.1117/1.JMM.21.1.014401]

Keywords: extreme ultraviolet; pellicle; wrinkle; coefficient of thermal expansion; critical dimension.

Paper 21092G received Nov. 14, 2021; accepted for publication Jan. 10, 2022; published online Feb. 1, 2022.

\section{Introduction}

Even though the extreme ultraviolet transmittance (EUVT) and thermal stability of EUV pellicles have been improved, it is necessary to confirm the effect on the imaging performance when a EUV pellicle is employed in the lithography process. ${ }^{1}$ As the temperature of the EUV pellicle increases under EUV exposure, the corresponding slit area expands and forms a wrinkle. ${ }^{1,2}$ This wrinkle induces EUVT nonuniformity because of the different EUV-light paths through the wrinkled EUV pellicle, ultimately impacting the imaging performance. ${ }^{3}$ It was reported that, because of the wrinkles formed in the EUV pellicle during the exposure, the critical dimension uniformity (CDU) impact can be limited to $0.1 \mathrm{~nm}$ by maintaining the local tilt angle below $300 \mathrm{mrad}^{4}{ }^{4}$ Although the value of the local tilt angle has been specified, the corresponding thermomechanical property guidelines have not been reported. ${ }^{5}$ Previous studies assumed a twodimensional wrinkle profile and an arbitrary period independent of the material properties of the EUV pellicle., ${ }^{2,6}$

*Address all correspondence to Jinho Ahn, jhahn@hanyang.ac.kr 


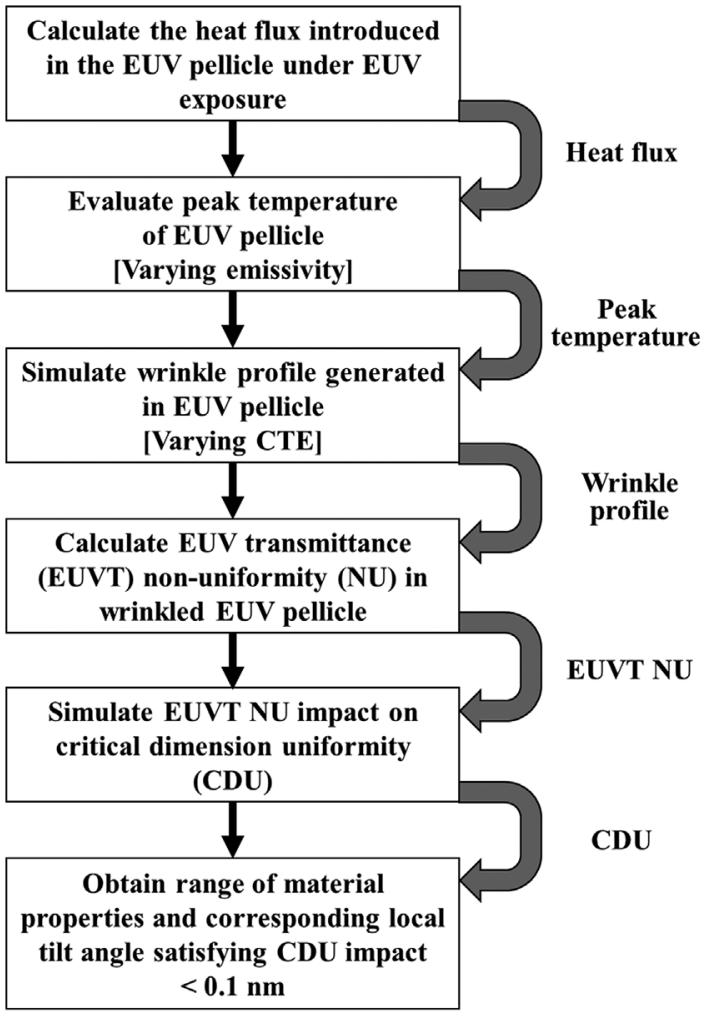

Fig. 1 Analysis flow for evaluating the ranges of the emissivity and CTE and the limit of the local tilt angle of the EUV pellicle for a CDU impact below $0.1 \mathrm{~nm}$.

We performed experimental, numerical, and finite element method (FEM) analyses to present the guidelines for the thermomechanical properties of the EUV pellicle according to the CDU impact specifications. ${ }^{7}$ The wrinkle profile of the pellicle was studied under EUV exposure, and the imaging performance was simulated using a wrinkled EUV pellicle under various values of emissivity and coefficients of thermal expansion (CTE) (Fig. 1). We present the guidelines of thermomechanical properties for the next-generation EUV pellicles, which meet the CDU specification.

\section{Experimental Methods}

\subsection{Experimental and Numerical Analyses for Evaluating the Peak Temperature of the Pellicle Under EUV Exposure}

Silicon-rich silicon nitride $\left(\mathrm{Si}_{6} \mathrm{~N}_{7}\right)$ was used as the EUV pellicle for experimental analysis to confirm the feasibility of the numerical and FEM analyses. ${ }^{8}$ Figure 2(a) shows the transmission electron microscopy (TEM) cross-sectional image of a 19-nm-thick $\mathrm{Si}_{6} \mathrm{~N}_{7}$ EUV pellicle, which exhibits $90 \%$ single-pass transmittance at a wavelength of $13.5 \mathrm{~nm}$. ${ }^{9}$ The thickness of $\operatorname{Si}_{6} \mathrm{~N}_{7}$ EUV pellicle used for subsequent analysis was extracted from the TEM result.

Figure 2(b) shows the schematic of the heat-load test apparatus used to emulate the thermal load during EUV scanning. ${ }^{10}$ The heat flux of the 355-nm UV laser can be matched with that of the EUV using Eq. (1), considering the absorbance of the pellicle at two different wavelengths. ${ }^{11}$ The absorbance $(0.1)$ at a wavelength of $13.5 \mathrm{~nm}$ was used for the 19 -nm-thick $\mathrm{Si}_{6} \mathrm{~N}_{7}$ pellicle according to the center for $\mathrm{x}$-ray optics database. ${ }^{12} \alpha_{355 \mathrm{~nm}}$ was set to 0.0344 based on the spectrophotometer results, and $A_{355 \mathrm{~nm}}$ was set to $0.283 \mathrm{~cm}^{2}$, corresponding to the area of a 0.6-cm-diameter beam. A two-channel pyrometer was used to eliminate the effect of emissivity changes during the measurement of the EUV pellicle temperature: ${ }^{13,14}$ 
(a)

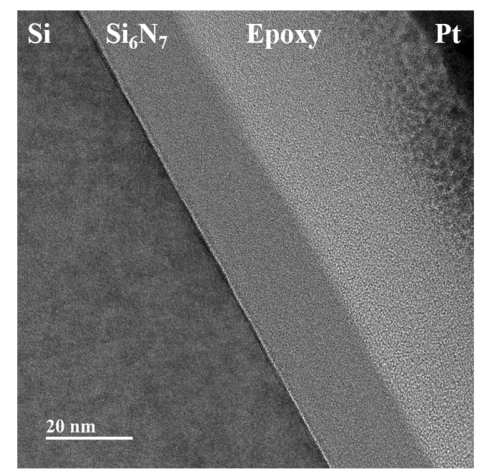

(b)

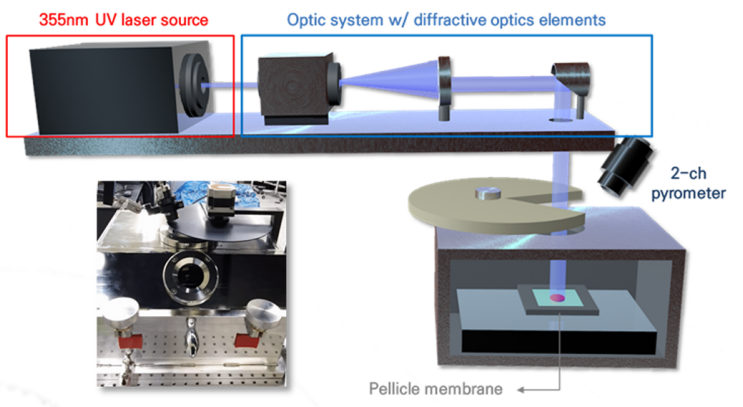

Fig. 2 (a) TEM cross-sectional image of the 19-nm-thick $\mathrm{Si}_{6} \mathrm{~N}_{7}$ EUV pellicle used for experimental analysis and (b) schematic of the heat-load test apparatus.

Table 1 Material properties and exposure conditions for calculating the peak temperature of the $\mathrm{Si}_{6} \mathrm{~N}_{7}$ EUV pellicle.

\begin{tabular}{llc}
\hline \hline Index & \multicolumn{1}{c}{ Contents } & Value \\
\hline$t$ & Exposure time (s) & 0.1 \\
$P$ & Incident EUV power in the slit area (W) & 55 \\
$m$ & Mass (kg) & $4.358 \times 10^{-9}$ \\
$\alpha$ & Absorbance & 0.1 \\
$C$ & Specific heat $\left(\mathrm{J} \mathrm{kg}^{-1} \mathrm{~K}^{-1}\right)$ & 673 \\
$\varepsilon$ & Emissivity & 0.0035 \\
$\sigma_{\mathrm{SB}}$ & Stefan-Boltzmann constant $\left(\mathrm{W} \mathrm{m}^{-2} \mathrm{~K}^{-4}\right)$ & $5.67 \times 10^{-8}$ \\
$S$ & Radiating area $\left(\mathrm{m}^{2}\right)$ & 0.0011 \\
$T_{s}$ & Surrounding temperature $(\mathrm{K})$ & 295.15 \\
\hline \hline & $\quad \alpha_{355 \mathrm{~nm}} \frac{P_{355 \mathrm{~nm}}}{A_{355 \mathrm{~nm}}}=\alpha_{13.5 \mathrm{~nm}} \frac{P_{13.5 \mathrm{~nm}}}{A_{13.5 \mathrm{~nm}}}$
\end{tabular}

where $\alpha$ is the absorbance, $P$ is the power, $A$ is the beam size, and each subindex is the wavelength of the light source.

The change in the temperature of the EUV pellicle over time $(d T / d t)$, with the varying the emissivity and specific heat, was calculated using the Stefan-Boltzmann equation, considering radiation to be the only heat dissipation mechanism [Eq. (2)]., ${ }^{2,15,16}$ The material properties of the EUV pellicle and the exposure conditions for numerical analysis were considered to be independent of the temperature and were approximated by rounding to the fourth decimal place (Table 1): $2,17,18$

$$
\frac{d T}{d t}=\frac{1}{\mathrm{C} \cdot m}\left[\alpha \cdot P-\varepsilon \cdot \sigma_{S B} \cdot S \cdot\left(T^{4}-T_{s}^{4}\right)\right]
$$

\subsection{FEM Simulation of a Wrinkle in the Slit Area of the EUV Pellicle}

Several studies performed FEM analysis using the ANSYS workbench to evaluate the wrinkles in the membrane. ${ }^{19-22}$ We also used ANSYS 2021R1 to simulate the wrinkle profile in a fullsized $(110 \mathrm{~mm} \times 143 \mathrm{~mm})$ pellicle under EUV exposure. ${ }^{23}$ 
The simulation was performed using steady-state thermomechanically coupled analysis. The experimental results were compared with the FEM results using the parameters in Table 1 to confirm the effectiveness of the FEM thermal analysis. The temperature of the pellicles under EUV scanning was calculated using the conditions in Table 1, except for the exposure time $(0.01 \mathrm{~s})$ and incident heat flux $\left(7.925 \mathrm{~W} \mathrm{~cm}^{-2}\right.$ considering $65 \%$ reflection from the mask). ${ }^{2,24-26}$ The thermal analysis assumed uniform heat flux in a $10 \mathrm{~mm} \times 110 \mathrm{~mm}$ rectangular slit and radiation as the only mechanism for heat dissipation. Tensile residual stress and gravity were applied to the EUV pellicle before applying the temperature load during the structural analysis. $^{27}$ The wrinkle profile of the EUV pellicle was simulated at different emissivity values $(0.025,0.05,0.1,0.2$, and 0.4$)$ and CTEs $\left(0.5 \times 10^{-5}, 1 \times 10^{-5}, 2 \times 10^{-5}\right.$, and $\left.4 \times 10^{-5} \mathrm{~K}^{-1}\right){ }^{17,28-31}$ The CTE range was set according to the change in the CTE due to heating and oxidation. ${ }^{32-37}$ The EUV pellicle deformation was assumed to be elastic, and nonlinear analysis was performed for large deflections. ${ }^{38}$

\subsection{Numerical Analysis of the EUV-Light Path and EUVT Non-Uniformity in a Wrinkled EUV Pellicle}

The biaxial wrinkle profile in the rectangular plate used for numerical modeling ${ }^{39}$ is given by the following equation:

$$
w(x, y)=A\left(\sin \frac{m \pi}{a} x\right)^{4}\left(\sin \frac{n \pi}{b} y\right)^{3},
$$

where $w$ is the out-of-plane deformation in the $Z$ direction at the $(x, y)$ coordinate, $A$ is the wrinkle amplitude, $m$ and $n$ are the number of half-wavelengths in the $X$ and $Y$ directions, and $a$ and $b$ are the lengths of the slit in the $X$ direction $(10 \mathrm{~mm})$ and $Y$ direction $(110 \mathrm{~mm})$, respectively.

The number of half-wavelengths and the average wrinkle amplitude of the simulated results were substituted in Eq. (3). Wrinkles within $3 \mathrm{~mm}$ of the border were excluded as they did not affect the imaging performance owing to the black border of the mask, ${ }^{40}$ and the modeled wrinkle profiles were plotted as a $10 \mathrm{~mm} \times 100 \mathrm{~mm} X Y$-plane contour graph with a grid size of $100 \mu \mathrm{m}$.

The EUV-light path length through the wrinkled pellicle, calculated using MATLAB R2020b, was used to evaluate the EUVT nonuniformity of the wrinkled region in the EUV pellicle. The single-pass EUVT through the pellicle membrane was calculated using Eqs. (4) and $(5)^{41}$ with fixed values of the refractive index $\eta_{p}(0.983)$ and extinction coefficient $\kappa_{p}$ (0.006) at wavelength $\lambda(13.5 \mathrm{~nm})$. The length of the EUV-light path through the wrinkled pellicle is $l$, and the proportional coefficient used for calculating the EUVT is $D$.

The effect of wrinkles on the imaging performance was evaluated using a double-pass EUVT, which is the product of the EUVT in the first and second passes, and the EUVT nonuniformity was calculated as the difference between the maximum and minimum double-pass EUVT in the slit area: ${ }^{3}$

$$
\begin{gathered}
\operatorname{EUVT}=\frac{8\left(\eta_{p}^{2}+\kappa_{p}^{2}\right)}{D} . \\
D=\left[\left(\eta_{p}^{2}+\kappa_{p}^{2}+1\right)^{2}+4 \eta_{p}^{2}\right] \cosh \left(\frac{4 \pi \kappa_{p} l}{\lambda}\right)+4 \eta_{p}\left(\eta_{p}^{2}+\kappa_{p}^{2}+1\right) \sinh \left(\frac{4 \pi \kappa_{p} l}{\lambda}\right) \\
-\left[\left(\eta_{p}^{2}+\kappa_{p}^{2}-1\right)^{2}-4 \kappa_{p}^{2}\right] \cos \left(\frac{4 \pi \kappa_{p} l}{\lambda}\right)+4 \kappa_{p}\left(\eta_{p}^{2}+\kappa_{p}^{2}-1\right) \sin \left(\frac{4 \pi \kappa_{p} l}{\lambda}\right) .
\end{gathered}
$$

\subsection{CDU Impact Due to the Double-Pass EUVT Nonuniformity in a Wrinkled EUV Pellicle}

The CDU impact of the wrinkles in the EUV pellicle during patterning was simulated using Panoramic Hyperlith, and the parameters used for imaging performance simulation are listed 
Table 2 Parameters of the mask, illumination, and EUV pellicle used for imaging performance simulation.

\begin{tabular}{lc}
\hline \hline Contents & Value \\
\hline Mask absorber material/thickness & TaBN/55 nm \\
Refractive index of the absorber $\left(\eta_{\mathrm{ab}}\right)$ & 0.95 \\
Extinction coefficient of the absorber $\left(\kappa_{\mathrm{ab}}\right)$ & 0.031 \\
Critical dimension (pitch) of $L / S(\mathrm{~nm})$ & $17(34), 16(32), 15(30)$ \\
Illumination condition $\sigma_{\text {inner }} / \sigma_{\text {outer }}$ & Dipole $0.1 / 0.8$ \\
Multilayer reflectivity & $65 \%$ \\
EUV pellicle material & $\mathrm{Si}_{6} \mathrm{~N}_{7}$ \\
Refractive index of the pellicle $\left(\eta_{p}\right)$ & 0.983 \\
Extinction coefficient of the pellicle $\left(\kappa_{p}\right)$ & 0.006 \\
\hline \hline
\end{tabular}

in Table 2. ${ }^{12,42,43}$ The refractive index and extinction coefficient were approximated by rounding to the fourth decimal place. The aerial images through pellicles with different EUVT were simulated separately for the 17, 16, and $15 \mathrm{~nm}$ half-pitch (HP) L/S patterns because Hyperlith cannot accommodate the wrinkles in the EUV pellicle. The threshold intensity that meets the target CD without the pellicle ("no pellicle" condition) was used as the reference intensity, and the CD after double-pass through the EUV pellicle was obtained. The CDU impact owing to the double-pass EUVT in the wrinkled EUV pellicle was then calculated using the derived equation. ${ }^{3}$

\section{Results and Discussion}

\subsection{Peak Temperature of the EUV Pellicle at Various Emissivity and Specific Heat Values}

The peak temperatures obtained from the numerical and FEM analyses were compared with the experimental values obtained by irradiating the pellicle using a $355-\mathrm{nm}$ laser equivalent to $5 \mathrm{~W} \mathrm{~cm}^{-2}$ EUV for $0.1 \mathrm{~s}$ [Fig. 3(a)]. The average peak temperature of the EUV pellicle $(1752 \mathrm{~K})$ measured by the heat load apparatus is consistent with the results of the numerical $(1830 \mathrm{~K})$ and FEM analyses $(1834 \mathrm{~K})$. The small deviation $(4.5 \%)$ from the experimental data confirms that the numerical and FEM analyses can be used for calculating the peak temperature of the pellicle.

The peak temperature was calculated while varying the emissivity and specific heat of the EUV pellicle under the double-pass with an EUV incident power of $5 \mathrm{~W} \mathrm{~cm}^{-2}$ and a $65 \%$ mask reflection [Figs. 3(b) and 3(c)]. The average peak temperature decreases by 15\%,34\%, 56\%, and $82 \%$ as the emissivity increased by a factor of $2,4,8$, and 16 from 0.025 , respectively. The average peak temperature decreases by $1.3 \%$ and $4.3 \%$ with a two- and three-factor increase in the specific heat, respectively. The density of potential EUV pellicle materials ranges from 1000 to $6000 \mathrm{~kg} \mathrm{~m}^{-3}$, limiting the effect of the specific heat on the peak temperature. ${ }^{44-46}$ Consequently, only the emissivity was considered during the analysis of the thermal properties of the EUV pellicle.

\subsection{FEM Analysis of the Wrinkle Profile and Numerical Analysis of the Double-Pass EUVT Nonuniformity in the Slit Area}

Figure 4(a) shows the isometric view of the wrinkle profile in the exposed slit area when EUV heat flux of $7.925 \mathrm{~W} \mathrm{~cm}^{-2}$ is applied to the center of the 19-nm-thick EUV pellicle for $10 \mathrm{~ms}$. 
(a)

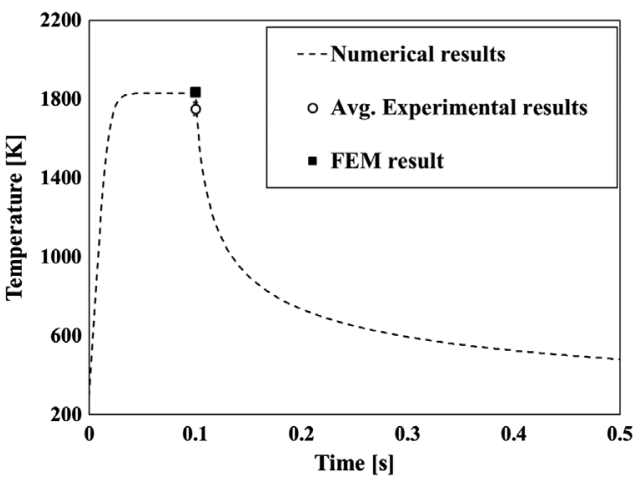

(c)

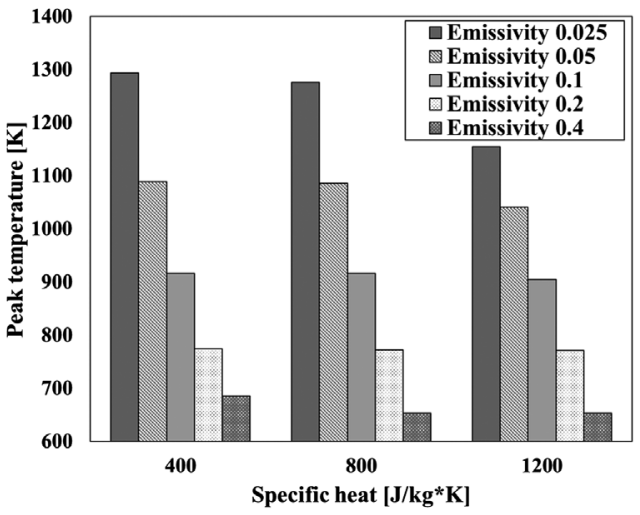

(b)

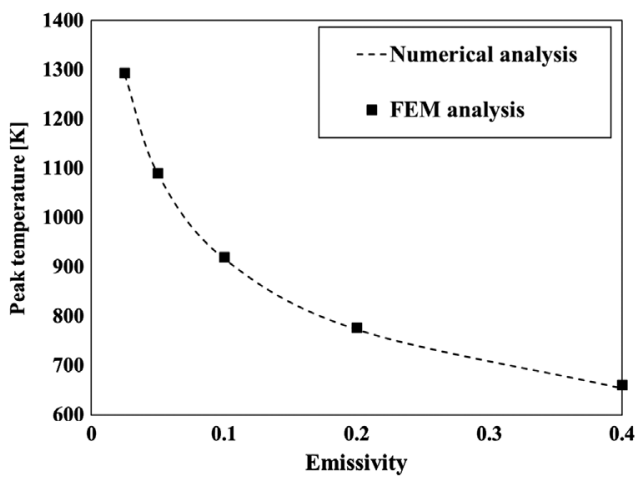

Fig. 3 (a) Peak temperatures of the experimental, numerical, and FEM analyses when $5 \mathrm{~W} \mathrm{~cm}^{-2}$ heat flux is introduced in the 19-nm-thick $\mathrm{Si}_{6} \mathrm{~N}_{7}$ EUV pellicle for $100 \mathrm{~ms}$, (b) results of the numerical and FEM analyses when $7.925 \mathrm{~W} \mathrm{~cm}-2$ heat flux is introduced for $10 \mathrm{~ms}$ with $673 \mathrm{~J} \mathrm{~kg}^{-1} \mathrm{~K}^{-1} \mathrm{spe}$ cific heat and various emissivity, and (c) results of numerical analysis for the influence of the specific heat on the peak temperature of the pellicle.

The EUVT, emissivity, and CTE of the EUV pellicle were set to $90 \%, 0.1$, and $1 \times 10^{-5} \mathrm{~K}^{-1}$, respectively. When the applied compressive stress is higher than the critical buckling stress of the membrane, a wrinkle is formed along the direction of the applied tensile stress. ${ }^{47}$

Compressive stress is formed along the $X$ and $Y$ directions because of restrained thermal expansion due to the heat flux applied to the membrane. However, the compressive stresses along two directions are different as the magnitude of the compressive stress is proportional to the stiffness of the constraint. ${ }^{48}$ Although the compressive stress along the $X$ direction is low due to the freestanding membrane, the compressive stress along the $Y$ direction compensates for the residual stress of the membrane owing to the rigid border. Therefore, tensile stress is formed along the $X$ direction, while compressive stress is formed along the $Y$ direction, as shown in Fig. 4(a). ${ }^{47}$

The maximum wrinkle amplitude $(71.2 \mu \mathrm{m})$ was obtained at emissivity and CTE values of 0.025 and $4 \times 10^{-5} \mathrm{~K}^{-1}$, respectively, whereas the minimum amplitude $(15.6 \mu \mathrm{m})$ was obtained at emissivity and CTE values of 0.4 and $5 \times 10^{-6} \mathrm{~K}^{-1}$, respectively [Fig. 4(b)]. The attenuation ratios of the emissivity and the CTE of the wrinkle amplitude were calculated at an emissivity of 0.025 and CTE of $4 \times 10^{-5} \mathrm{~K}^{-1}$ [Figs. 4(c) and 4(d), respectively]. The wrinkle amplitude attenuates with the increase in the emissivity and decrease in the CTE. However, the attenuation ratios of the two properties are not compared at the same level because emissivity determines the peak temperature of the pellicle, whereas the CTE determines the wrinkle profiles in the pellicle under temperature gradients. However, an appropriate combination of the emissivity and CTE can yield a wrinkle profile satisfying the CD specifications.

The color bars in Fig. 5 represent the amount of deformation in the $Z$ direction at each grid coordinate while varying the CTE of the pellicle. The numerically modeled wrinkle profile was 
(a)

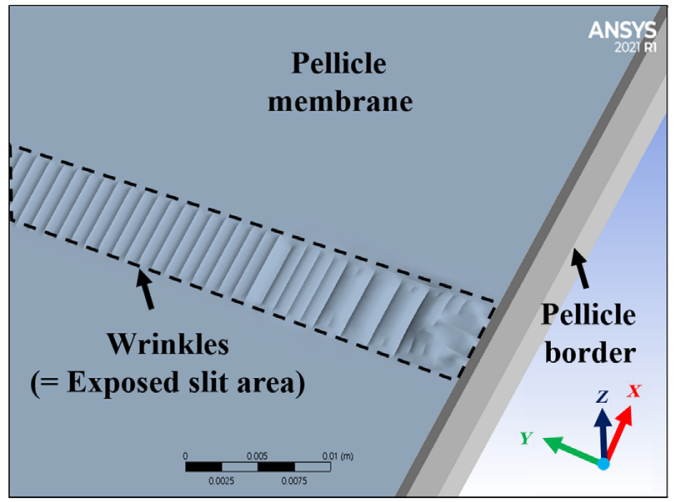

(c)

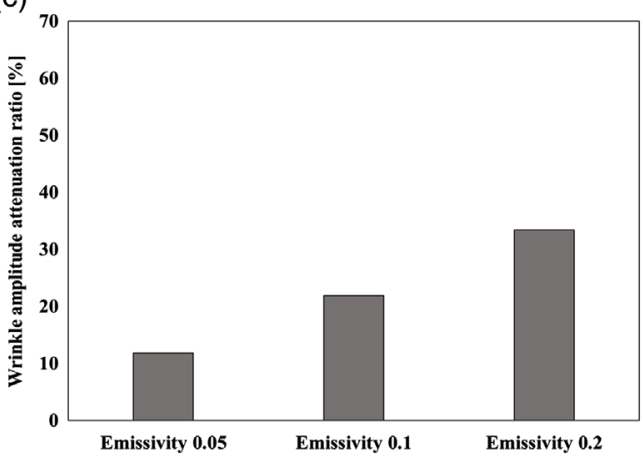

(b)

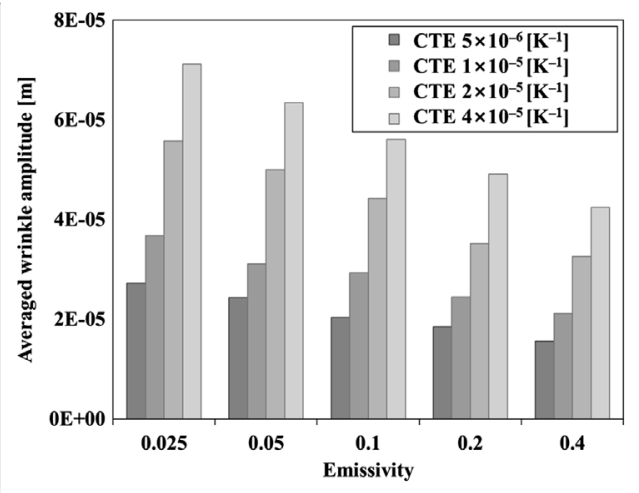

(d)

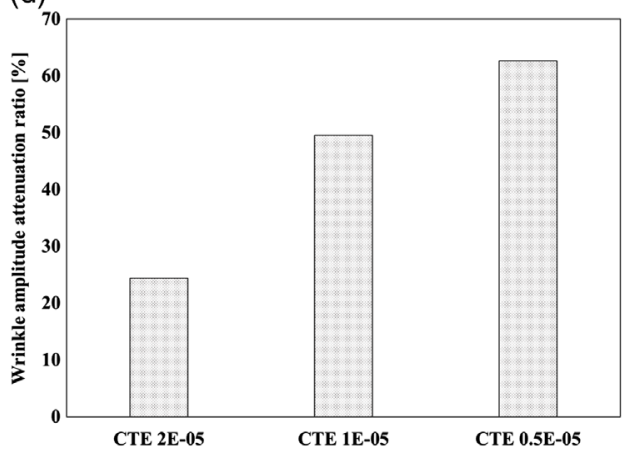

Fig. 4 (a) Total deformation results of the FEM analysis of 19-nm-thick EUV pellicles at an emissivity of 0.1 , CTE of $1 \times 10^{-5} \mathrm{~K}^{-1}$, and single-pass EUVT of $90 \%$ at $920.61 \mathrm{~K}$. (b) Results of the averaged wrinkle amplitude on varying the emissivity and CTE of the pellicle, and attenuation ratios of the (c) emissivity and (d) CTE for the wrinkle amplitude.

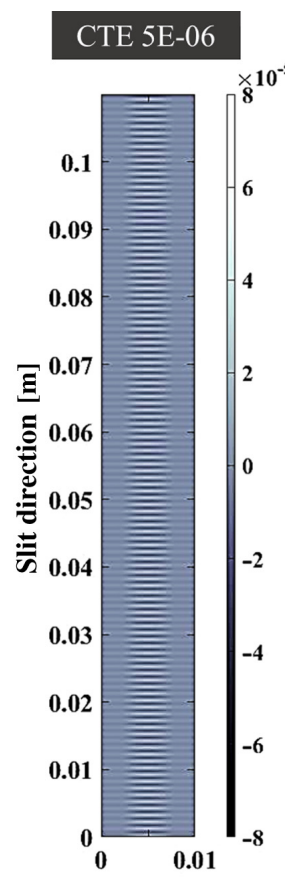

Scan direction $[\mathrm{m}]$

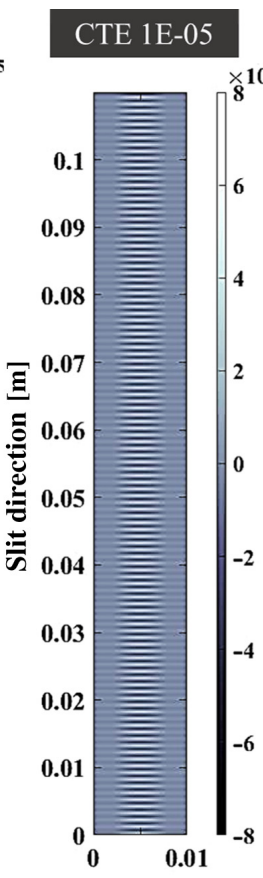

Scan direction $[\mathrm{m}]$

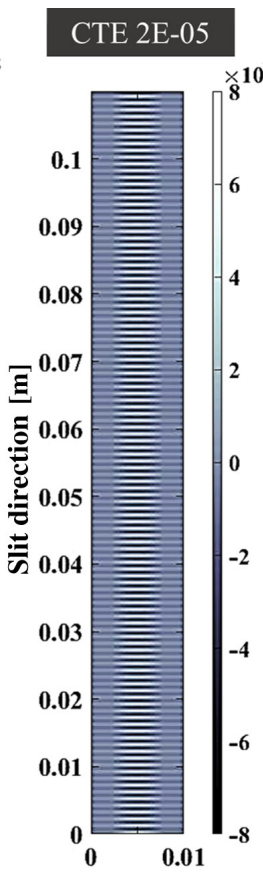

Scan direction $[\mathrm{m}]$

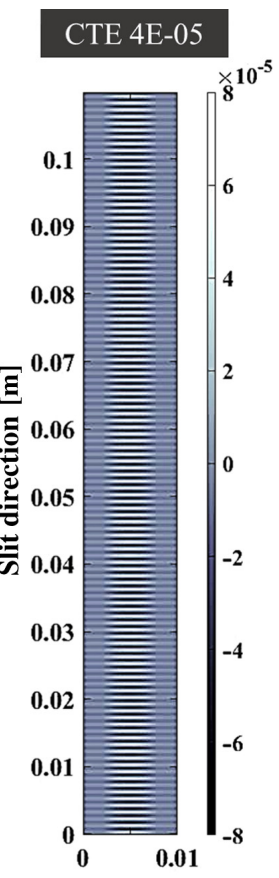

Scan direction $[\mathrm{m}]$

Fig. 5 Contour plot of the modeled wrinkle profiles using MATLAB R2020b when EUV pellicles, with a single-pass EUVT of $90 \%$, emissivity of 0.05 , and various CTEs $\left(5 \times 10^{-6}, 1 \times 10^{-5}, 2 \times 10^{-5}\right.$, and $4 \times 10^{-5} \mathrm{~K}^{-1}$ from left to right), are exposed to EUV heat flux of $7.925 \mathrm{~W} \mathrm{~cm}^{-2}$ for $10 \mathrm{~ms}$. 
(a)

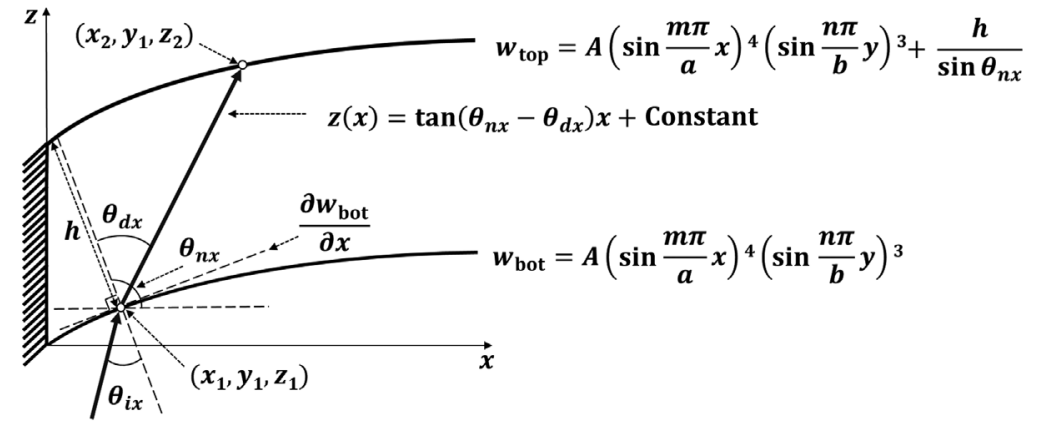

(b)

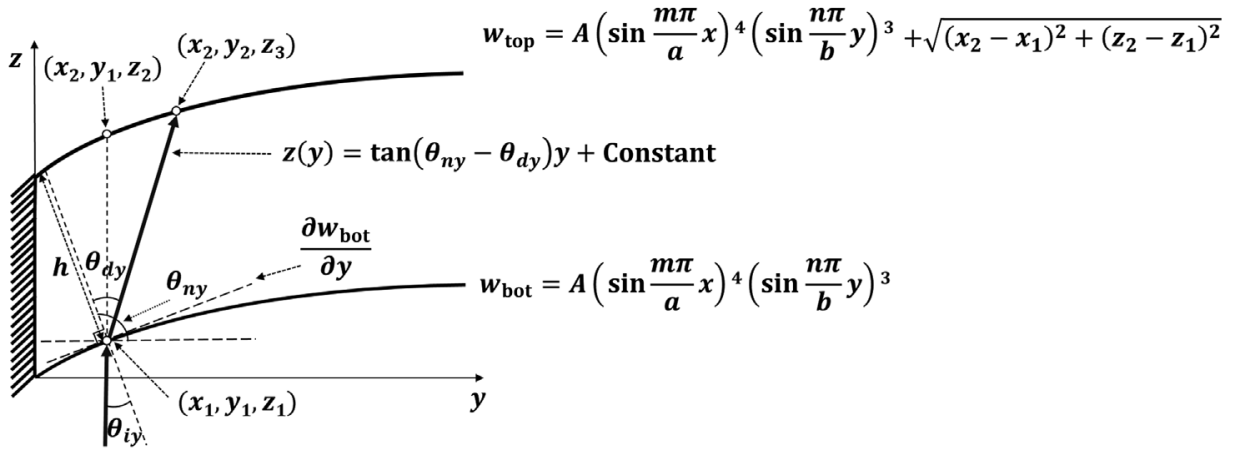

Fig. 6 (a) Schematic of the diffracted EUV-light path in the $X$ direction incident at 6 deg on the pellicle and (b) in the $Y$ direction incident normally on the pellicle.

used to calculate the EUV-light length through the wrinkled pellicle owing to its similarity with the simulation results.

Figure 6(a) shows the cross-section of the modeled wrinkle, which is divided by the $X Z$ plane, corresponding to an arbitrary $y_{1}$ on the wrinkled surface. The EUV-light path is denoted by a black arrow with a 6-deg chief ray angle at the object (CRAO) along the $X$ direction. ${ }^{49}$ The bottom surface ( $w_{\text {bot }}$ ) of the wrinkle is expressed using Eq. (3) and the top surface $\left(w_{\text {top }}\right)$ is the sum of $h / \sin \left(\theta_{n x}\right)\left(\theta_{n x}\right.$ is the slope of the line normal to the wrinkle bottom) and $w_{\text {bot }}$ because the perpendicular distance between $w_{\text {bot }}$ and $w_{\text {top }}$ denotes the EUV pellicle thickness $(h)$. The incident angle $\left(\theta_{i x}\right)$ of the EUV-light at $w_{\text {bot }}$ was calculated by subtracting $\theta_{n x}$ from the slope of the incident line with 6-deg CRAO (84 deg). The diffraction angle $\left(\theta_{d x}\right)$ was calculated using Eq. (6), which was derived from Snell's law, with the refractive indices of vacuum and the EUV pellicle set to 1 and $\eta_{p}$, respectively. ${ }^{50}$ The value of $x_{2}$ was calculated using Eq. (7), assuming that the EUV- light incident on the bottom of the wrinkle at $\left(x_{1}, y_{1}, z_{1}\right)$ passes through the pellicle and contacts the top surface at $\left(x_{2}, y_{1}, z_{2}\right)$ :

$$
\begin{aligned}
\theta_{d x}= & \sin ^{-1}\left(\frac{\sin \left(\tan ^{-1}\left(\frac{-1}{4 A \cdot \frac{m \pi}{a}\left(\cos \frac{m \pi}{a} x_{1}\right)\left(\sin \frac{m \pi}{a} x_{1}\right)^{3}\left(\sin \frac{n \pi}{b} y_{1}\right)^{3}}\right)-84 \mathrm{deg}\right)}{\eta_{p}}\right), \\
A \cdot & \left(\sin \left(\frac{n \pi}{b} y_{1}\right)\right)^{3}\left(\sin \left(\frac{m \pi}{a} x\right)\right)^{4}-\tan \left(\theta_{n x}-\theta_{d x}\right) x+\frac{h}{\sin \theta_{n x}} \\
& -A \cdot\left(\sin \left(\frac{n \pi}{b} y_{1}\right)\right)^{3}\left(\sin \left(\frac{m \pi}{a} x_{1}\right)\right)^{4}+\tan \left(\theta_{n x}-\theta_{d x}\right) x_{1}=0 .
\end{aligned}
$$

Figure 6(b) shows the cross-section of the wrinkled EUV pellicle divided by a plane, with a line in $w_{\text {bot }}$ when $x=x_{1}$ and a line in $w_{\text {top }}$ when $x=x_{2}$. The sum of $\sqrt{\left(x_{2}-x_{1}\right)^{2}+\left(z_{2}-z_{1}\right)^{2}}$ and $w_{\text {bot }}$ gives $w_{\text {top }}$. The diffraction angle of the $Y Z$-plane $\left(\theta_{d y}\right)$ was calculated by substituting the slopes of the line perpendicular to the bottom of the wrinkle $\left(\theta_{n y}\right)$ and that of the incident line $\left(\theta_{i y}\right)$ into Snell's law. Assuming that the EUV-light incident on the bottom at $\left(x_{1}, y_{1}, z_{1}\right)$ contacts 


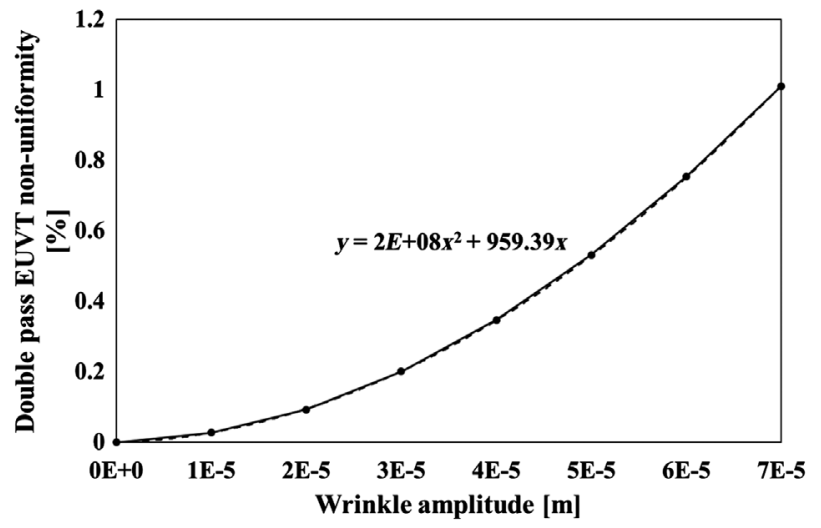

Fig. 7 Values of the double-pass EUVT nonuniformity according to the wrinkle amplitude. The dashed line represents the fitted polynomial curve.

the top of the wrinkle at $\left(x_{2}, y_{2}, z_{3}\right)$ due to refraction in the $X$ and $Y$ directions within the pellicle, the distance between the two points gives the EUV-light length within the wrinkled pellicle. Equation (8) was used to calculate $y_{2}$ and the single-pass EUVT was calculated by substituting the total length of the EUV-light in Eq. (4). Figure 7 shows the plot of double-pass EUVT nonuniformity according to the wrinkle amplitude:

$$
\begin{aligned}
& \mathrm{A}\left(\sin \left(\frac{m \pi}{a} x_{2}\right)\right)^{4}\left(\sin \left(\frac{n \pi}{b} y\right)\right)^{3}-\tan \left(\theta_{n y}-\theta_{d y}\right) y+\sqrt{\left(x_{2}-x_{1}\right)^{2}+\left(z_{2}-z_{1}\right)^{2}} \\
& -\mathrm{A}\left(\sin \left(\frac{m \pi}{a} x_{1}\right)\right)^{4}\left(\sin \left(\frac{n \pi}{b} y_{1}\right)\right)^{3}+\tan \left(\theta_{n y}-\theta_{d y}\right) y_{1}=0 .
\end{aligned}
$$

(a)

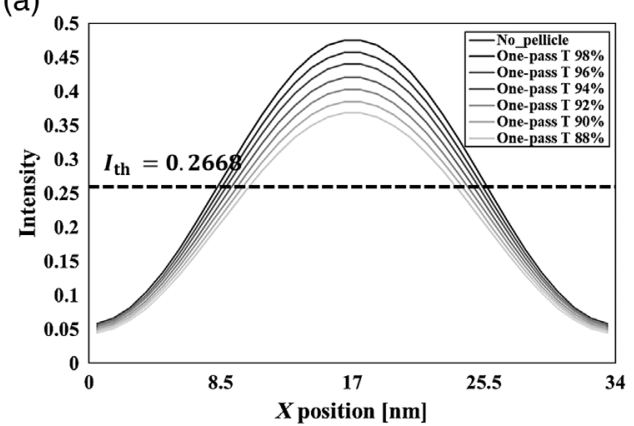

(c)

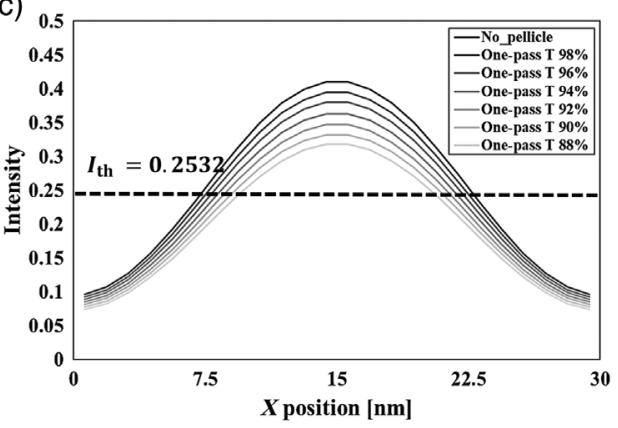

(b)

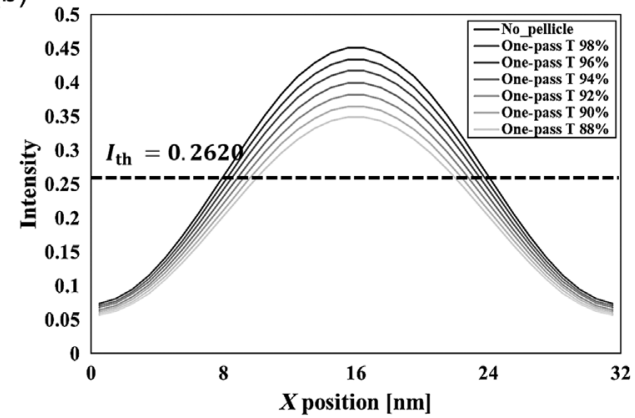

Fig. 8 Aerial images through the EUV pellicles with a single-pass EUVT of $88 \%$ to $98 \%$ for (a) $17 \mathrm{~nm}$, (b) $16 \mathrm{~nm}$, and (c) $15 \mathrm{~nm} \mathrm{HP} \mathrm{L/S} \mathrm{patterns.} \mathrm{The} \mathrm{dashed} \mathrm{line} \mathrm{represents} \mathrm{the} \mathrm{threshold}$ intensity $(0.2668,0.2620$, and 0.2532 for 17,16 , and $15 \mathrm{~nm} \mathrm{HP} \mathrm{L/S,} \mathrm{respectively)} \mathrm{under} \mathrm{the} \mathrm{"no}$ pellicle" condition. 


\subsection{Calculation of the CDU Impact as a Function of the Double-Pass EUVT Nonuniformity in the Pellicle}

Figures 8(a)-8(c) show the aerial images through the EUV pellicles with an $88 \%$ to $98 \%$ singlepass EUVT for the 17, 16, and $15 \mathrm{~nm}$ HP L/S patterns, respectively. It can be inferred from the results that the CDU depends on the EUVT of the EUV pellicle.

The $C D$ values at the threshold intensity were fitted linearly with the above equations [Fig. 9(a)]. The CDU impact depends on the nonuniformity of the double-pass EUVT in the pellicle, and the slopes corresponding to the 17, 16, and $15 \mathrm{~nm} \mathrm{HP} \mathrm{L/S} \mathrm{patterns} \mathrm{are} \mathrm{shown}$ in Fig. 9(b). The nonuniformity of the double-pass EUVT should be less than $0.4973 \%$,

(a)

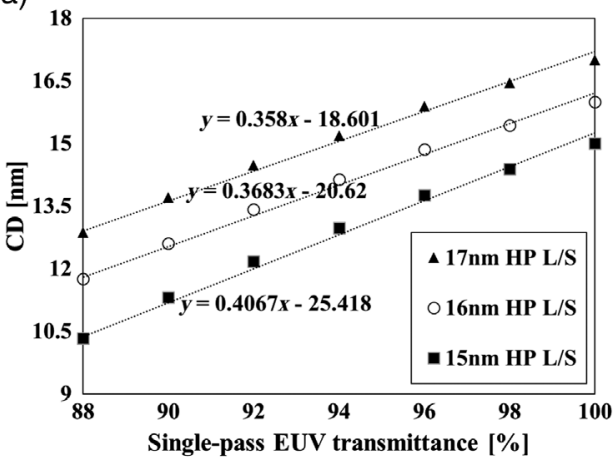

(b)

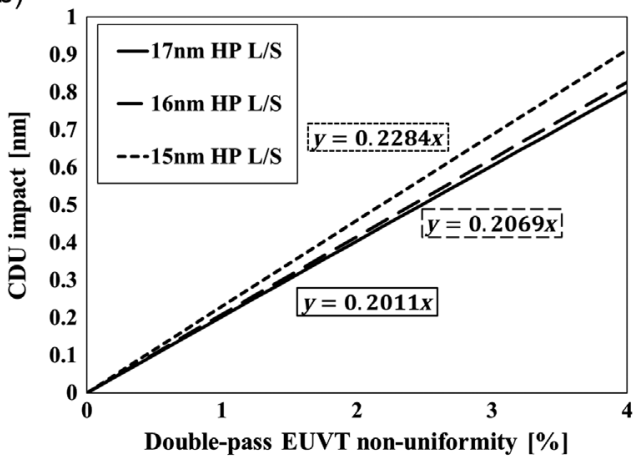

Fig. 9 (a) CD as a function of the single-pass EUVT of the pellicle for 17, 16, and $15 \mathrm{~nm} \mathrm{HP}$ L/S patterns and (b) CDU impact as a function of the double-pass EUVT nonuniformity calculated based on the single-pass EUVT of $88 \%$.

(a)

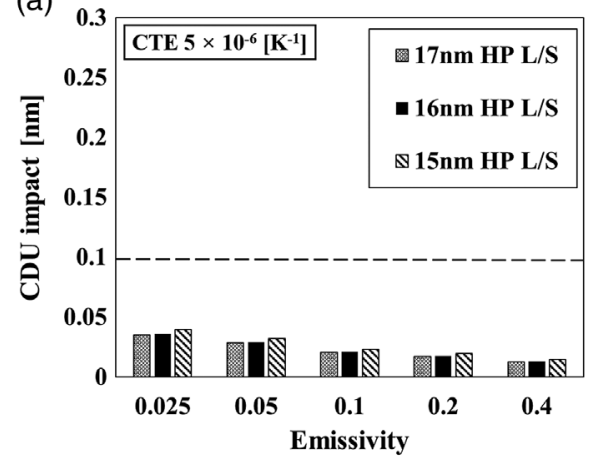

(c)

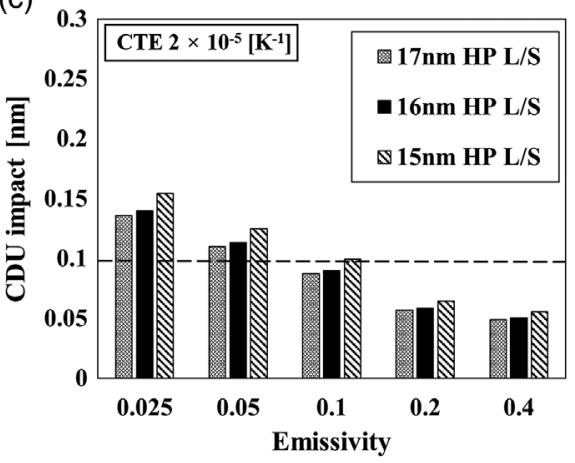

(b)

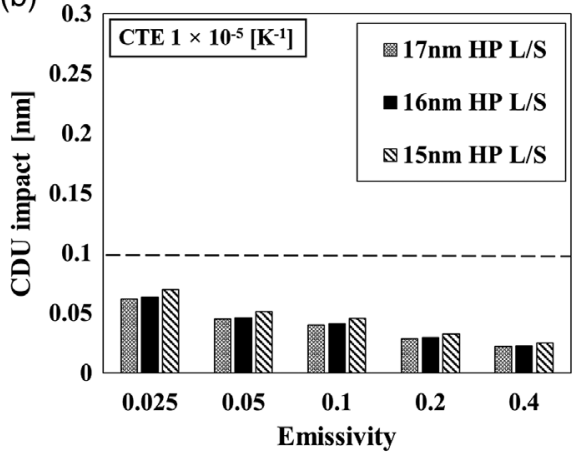

(d)

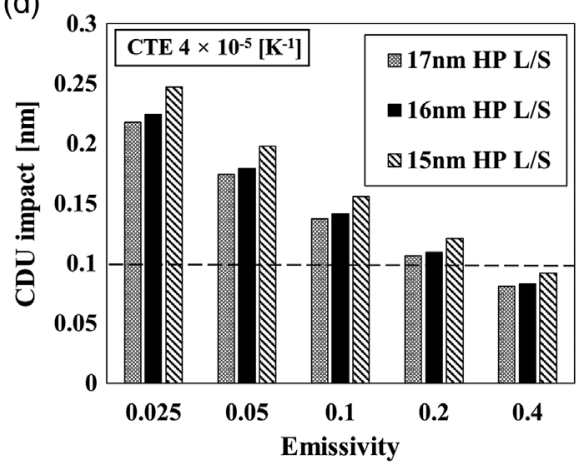

Fig. 10 Results of the CDU impact when EUV pellicles with an isotropic elastic modulus of $317 \mathrm{GPa}$, Poisson's ratio of 0.23 , refractive index of 0.9832 , and thickness of $19 \mathrm{~nm}$ with various emissivity values $(0.025,0.05,0.1,0.2$, and 0.4$)$ and CTEs $\left[(a) 5 \times 10^{-6}\right.$, (b) $1 \times 10^{-5}$, (c) $2 \times 10^{-5}$, and (d) $4 \times 10^{-5} \mathrm{~K}^{-1}$ ] are used for patterning of 17,16 , and $15 \mathrm{~nm} \mathrm{HP}$ L/S. 
$0.4833 \%$, and $0.4378 \%$ for the 17,16 , and $15 \mathrm{~nm} \mathrm{HP} \mathrm{L/S} \mathrm{patterns,} \mathrm{respectively,} \mathrm{for} \mathrm{a} \mathrm{CDU}$ impact below $0.1 \mathrm{~nm}$.

\subsection{Calculation of the Local Tilt Angle in the EUV Pellicle and Material Property Guidelines for the EUV Pellicle to Meet the CDU Impact Specifications}

The maximum local tilt angle $\left(\theta_{\max }=\tan ^{-1}(A \cdot 2 \pi / 2 \cdot\right.$ length of half wavelength $\left.)\right)$ of the EUV pellicle was calculated according to the $\mathrm{CD}$ specifications. ${ }^{2}$ The maximum local tilt angles for 17, 16, and $15 \mathrm{~nm} \mathrm{HP} \mathrm{L/S} \mathrm{are} \mathrm{290.2,} \mathrm{286.1,} \mathrm{and} 272.3 \mathrm{mrad}$, corresponding to wrinkle amplitudes of $47.5,46.8$, and $44.4 \mu \mathrm{m}$, respectively.

The dashed line in Fig. 10 represents the CDU impact of $0.1 \mathrm{~nm} .{ }^{7}$ EUV pellicles with a CTE of $1 \times 10^{-5} \mathrm{~K}^{-1}$ or less satisfy the CD specification with a CDU impact of 0.013 to $0.07 \mathrm{~nm}$ in the emissivity range of 0.025 to 0.4 . However, an EUV pellicle with a CTE of $2 \times 10^{-5} \mathrm{~K}^{-1}$ and emissivity of 0.1 has a CDU impact of $0.0992 \mathrm{~nm}$ during $15-\mathrm{nm}$ HP L/S patterning. The emissivity should be greater than 0.4 for a pellicle with a CTE of $4 \times 10^{-5} \mathrm{~K}^{-1}$ to meet the CD specification during 15 to $17 \mathrm{~nm} \mathrm{HP}$ L/S patterning. For example, an EUV pellicle with a 3-nm ruthenium capping layer, which exhibits an emissivity of 0.4 , cannot be used for an HP L/S pattern below $15 \mathrm{~nm}$ if the CTE of the EUV pellicle exceeds $4 \times 10^{-5} \mathrm{~K}^{-1}{ }^{29}$ The CDU impact specifications are satisfied when EUV pellicles with a CTE of $2 \times 10^{-5} \mathrm{~K}^{-1}$ or less and emissivity of 0.1 or more are used during 15 to $17 \mathrm{~nm} \mathrm{HP} \mathrm{L/S} \mathrm{patterning.}$

\section{Conclusion}

In this study, the peak temperature of the EUV pellicle during EUV exposure was evaluated as a function of its emissivity through experimental, numerical, and FEM analyses. In addition, the wrinkle profiles of the pellicles were modeled in three dimensions by varying the CTE. The CDU impact due to EUVT nonuniformity in the wrinkled EUV pellicle was calculated, and the emissivity and CTE range for the pellicle were calculated to satisfy the CD specifications.

Although emissivity increases the thermal stability of the EUV pellicle by lowering its peak temperature, the CTE of the pellicle should also be considered to satisfy the CDU impact specifications during exposure. These guidelines for the emissivity and CTE of EUV pellicles can be extended to next-generation EUV pellicles through subsequent studies on other materials.

\section{Acknowledgments}

This research was supported by the Nano Material Technology Development Program of the National Research Foundation of Korea (NRF) funded by the Ministry of Education, Science and Technology (No. 2019K1A3A1A14067316), the Technology Innovation Program (No. 20009803, Development of pellicle material for EUV process) funded by the Ministry of Trade, Industry and Energy, and the BK21 FOUR (Fostering Outstanding University for Research) program through the National Research Foundation (NRF) funded by the Ministry of Education of Korea (5199991614716). The authors declare that there is no conflict of interest.

\section{References}

1. B. Wu and A. Kumar, "Extreme ultraviolet lithography: a review," J. Vac. Sci. Technol. B: Microelectron. Nanometer Struct. Process. Meas. Phenom. 25(6), 1743-1761 (2007).

2. D. L. Goldfarb, M. O. Bloomfield, and M. Colburn, "Thermomechanical behavior of EUV pellicle under dynamic exposure conditions," Proc. SPIE 9776, 977621 (2016).

3. I.-S. Kim et al., "Impact of transmission non-uniformity of a wrinkled EUV pellicle for N5 patterning under various illuminations," Microelectron. Eng. 177, 35-40 (2017). 
4. C. Zoldesi et al., "Progress on EUV pellicle development," Proc. SPIE 9048, 90481N (2014).

5. H.-G. Shin and H.-K. Oh, "Extreme-ultraviolet pellicle durability comparison for better lifetime," Proc. SPIE 11147, 111470U (2019).

6. I.-S. Kim et al., "Impact of a deformed extreme ultraviolet pellicle in terms of the critical dimension uniformity," J. Micro/Nanolithogr. MEMS MOEMS 15(2), 021003 (2016).

7. L. Scaccabarozzi et al., "Investigation of EUV pellicle feasibility," Proc. SPIE 8679, 867904 (2013).

8. J. E. Kim et al., "Manufacturing SiNx extreme ultraviolet pellicle with HF wet etching process," J. Semicond. Disp. Technol. 14(3), 7-11 (2015).

9. J. H. Kim et al., "Manufacturing large-scale SiNx EUV pellicle with water bath," J. Semicond. Disp. Technol. 15(1), 17-21 (2016).

10. I. Pollentier et al., "The EUV CNT pellicle: balancing material properties to optimize performance," Proc. SPIE 11323, 113231G (2020).

11. Y. J. Jang et al., "Investigation of the mechanical/thermal properties of nano-scale silicon nitride membranes," J. Korean Inst. Met. Mater. 57(2), 124-129 (2019).

12. B. L. Henke, E. M. Gullikson, and J.C. Davis, "X-ray interaction: photoabsorption, scattering, transmission, and reflection at E $=50-30000 \mathrm{eV}, \mathrm{Z}=1-92$," At. Data Nucl. Data Tab. 54(2), 181-342 (1993).

13. M. Kulish et al., "The liner brightness temperature measurement by two channel optical pyrometer," J. Phys.: Conf. Ser. 946, 012042 (2018).

14. D. Youchison et al., "High heat flux testing of a helium-cooled tungsten tube with porous foam," Fusion Eng. Des. 82(15-24), 1854-1860 (2007).

15. P. Van Zwol et al., "Pellicle films supporting the ramp to HVM with EUV," Proc. SPIE 10451, 1045100 (2017).

16. H.-C. Lee et al., "Temperature behavior of pellicles in extreme ultraviolet lithography," J. Korean Phys. Soc. 61(7), 1093-1096 (2012).

17. C. H. Mastrangelo, Y.-C. Tai, and R. S. Muller, "Thermophysical properties of low-residual stress, silicon-rich, LPCVD silicon nitride films," Sens. Actuators A 23(1-3), 856-860 (1990).

18. A. Kaushik, H. Kahn, and A. H. Heuer, "Wafer-level mechanical characterization of silicon nitride MEMS," J. Microelectromech. Syst. 14(2), 359-367 (2005).

19. J. Leifer and W. Belvin, "Prediction of wrinkle amplitudes in thin film membranes using finite element modeling," in 44th AIAA/ASME/ASCE/AHS/ASC Struct. Struct. Dyn. and Mater. Conf. (2003).

20. C. Wang and X. Du, "Wrinkle analysis of space membrane structures and applications," Int. J. Comput. Methods Eng. Sci. Mech. 8(3), 159-164 (2007).

21. R. Ma, J. Zhang, and Q.-S. Yang, "An analysis on transition of membrane wrinkles applying shell elements," Eng. Mech. 28(8), 70-76 (2011).

22. J. Gao et al., "Wrinkling of thin membrane under thermal loading," in ASME 2006 Int. Mech. Eng. Congr. and Expos., pp. 479-485 (2006).

23. H.-H. Lee, Finite Element Simulations with ANSYS Workbench 2021: Theory, Applications, Case Studies, SDC Publications (2021).

24. M. Singh and J. J. Braat, "Design of multilayer extreme-ultraviolet mirrors for enhanced reflectivity," Appl. Opt. 39(13), 2189-2197 (2000).

25. M. Lowisch et al., "Optics for EUV production," Proc. SPIE 7636, 763603 (2010).

26. M. A. Soto and R. Venkatasubramanian, "ANSYS-based detailed thermo-mechanical modeling of complex thermoelectric power designs," in ICT 2005. 24th Int. Conf. Thermoelectr., 2005, pp. 219-221 (2005).

27. J. Kim, H. Kim, and J. Ahn, "Impact of residual stress on the deflection of extreme ultraviolet pellicles," J. Micro/Nanopatterning Mater. Metrol. 20(2), 024401 (2021).

28. S. Gusev et al., "Thermal stability of a freestanding EUV filter under long-term vacuum annealing at $700-1000^{\circ}$ C," J. Surf. Investig.: X-ray Synchrotron Neutron Tech. 6(3), 482-486 (2012). 
29. P. Van Zwol et al., "Emissivity of freestanding membranes with thin metal coatings," J. Appl. Phys. 118(21), 213107 (2015).

30. J. Lasjaunias et al., "Low-temperature specific heat of $\mathrm{MoSi}_{2}$," Phys. Rev. B 37(17), 10364 (1988).

31. H. Ftouni et al., "Thermal conductivity of silicon nitride membranes is not sensitive to stress," Phys. Rev. B 92(12), 125439 (2015).

32. T. Retajczyk, Jr and A. Sinha, "Elastic stiffness and thermal expansion coefficients of various refractory silicides and silicon nitride films," Thin Solid Films 70(2), 241-247 (1980).

33. M. Fukuhara, K. Fukazawa, and A. Fukawa, "Physical properties and cutting performance of silicon nitride ceramic," Wear 102(3), 195-210 (1985).

34. O. Thomas et al., "Molybdenum disilicide: crystal growth, thermal expansion and resistivity," Solid State Commun. 55(7), 629-632 (1985).

35. G. M. Jacobsen et al., "Fabrication and characterization of zirconium silicide for application to gas-cooled fast reactors," Nucl. Technol. 208, 1-10 (2021).

36. R. Hao et al., "Theoretical prediction of structural stability, electronic and elastic properties of $\mathrm{ZrSi}_{2}$ under pressure," RSC Adv. 5(46), 36779-36786 (2015).

37. A. Varadarajan, Thermomechanical Property Evaluation of Molybdenum Alloys, ProQuest, Ann Arbor (2004).

38. V. R. Kar and S. K. Panda, "Large deformation bending analysis of functionally graded spherical shell using FEM," Struct. Eng. Mech. 53(4), 661-679 (2015).

39. M.-H. Jen and Y.-H. Wu, "Wrinkling of extensional thin films through modified large deflection equations analytically and experimentally," AIP Adv. 5(12), 127239 (2015).

40. C. Turley et al., "EUV mask black border evolution," Proc. SPIE 9235, 923513 (2014).

41. L. N. Hadley and D. Dennison, "Reflection and transmission interference filters part I. theory," J. Opt. Soc. Am. 37(6), 451-465 (1947).

42. T. Shoki et al., "Process development of 6-in EUV mask with TaBN absorber," Proc. SPIE 4745, 857-864 (2002)

43. L. Devaraj et al., "Lithographic effects due to particles on high-NA EUV mask pellicle," Proc. SPIE 11177, 111770V (2019).

44. R. Huszank et al., "Determination of the density of silicon-nitride thin films by ion-beam analytical techniques (RBS, PIXE, STIM)," J. Radioanal. Nucl. Chem. 307(1), 341-346 (2016).

45. A. N. Christensen, "Crystal growth and characterization of the transition metal silicides $\mathrm{MoSi}_{2}$ and $\mathrm{WSi}_{2}$, , J. Cryst. Growth 129(1-2), 266-268 (1993).

46. D. R. Lide, CRC Handbook of Chemistry and Physics, CRC Press, Florida (2004).

47. S. P. Timoshenko and S. Woinowsky-Krieger, Theory of Plates and Shells, McGraw-Hill, New York (1959).

48. S. Xu, "Thermal stress analysis of dissimilar welding joints by finite element method," Procedia Eng. 15, 3860-3864 (2011).

49. X. Liu et al., "EUV source-mask optimization for $7 \mathrm{~nm}$ node and beyond," Proc. SPIE 9048 , 90480Q (2014).

50. C. Parazzoli et al., "Experimental verification and simulation of negative index of refraction using Snell's law," Phys. Rev. Lett 90(10), 107401 (2003).

Junghwan Kim received his bachelor's degree from the Department of Material Science and Engineering, Hanyang University (Seoul campus), Republic of Korea. Currently, he is pursuing the unified course of his master's and doctoral degrees at Hanyang University. His current research interests include the mechanics of materials for EUV pellicles and masks.

Haneul Kim received his bachelor's degree from the Department of Material Science and Engineering, Hanyang University (Seoul campus), Republic of Korea. Currently, he is pursuing the unified course of his master's and doctoral degrees at Hanyang University. His current research interests include the fabrication process of EUV pellicle and property measurement of EUV pellicle. 
Jinho Ahn received his BS and MS degrees from Seoul National University in 1986 and 1988, respectively, and his PhD from the University of Texas at Austin, in 1992, in materials science and engineering. He joined Hanyang University in 1995 as a professor of materials science and engineering. He has authored more than 160 papers and invented more than 50 patents. Currently, he is a director of the EUV-IUCC, a research center, to promote collaborative work for EUV lithography technology development. 